\title{
Current treatment options for meningioma
}

\author{
Caroline Apra ${ }^{a}$, Matthieu Peyre ${ }^{a, b}$ and Michel Kalamarides ${ }^{a, b}$ \\ aSorbonne Université, Paris, France; 'bepartment of Neurosurgery, Pitié Salpêtrière Hospital, Paris, France
}

\begin{abstract}
Introduction: With an annual incidence of 5/100,000, meningioma is the most frequent primary tumor of the central nervous system. Risk factors are radiotherapy and hormone intake. Most meningiomas are grade I benign tumors, but up to 15\% are atypical and 2\% anaplastic according to the WHO 2016 histological criteria.

Areas covered: This review details the current standard therapy based on international guidelines and recent literature, and describes new approaches developed to treat refractory cases. First-line treatments are observation and surgery, but adjuvant radiotherapy/radiosurgery is discussed for atypical and indicated for anaplastic meningiomas. The most problematic cases include skull base meningiomas that enclose vasculo-nervous structures and surgery- and radiation-refractory tumors that present with significant morbidity and mortality. The treatment of recurrent tumors is based on radiotherapy and repeated surgery. Systematic therapies are not effective in general but several clinical trials are ongoing. Expert commentary: Molecular characterization of the tumors, based on genetic mutations such as NF2, SMO, TERT, TRAF7, and on the methylation profile are developing, completing the histological classification and giving new insights into prognosis and treatment options.
\end{abstract}

KEYWORDS Intracranial tumor; neurosurgery; clinical trials; NF2; methylation

\section{Introduction}

Meningiomas arise from the meningeal coverings of the central nervous system. With an annual incidence of around 5 per 100,000 individuals, they are the most frequent primary tumors in the central nervous system (30\%) [1,2]. Meningiomas are diagnosed because of neurological symptoms (neurological deficits, epilepsy, elevated intracranial pressure) or can be discovered on brain imaging for unspecific unrelated symptoms, for instance tinnitus or headaches. Magnetic resonance imaging (MRI) are often sufficient to make the diagnosis and allow precise location and measurement of the tumor. The frequency of meningiomas is probably underestimated, since systematic screening MRI showed that the prevalence of undiagnosed meningiomas is $0.5 \%$ in the subjects aged $45-59$ years and $1.6 \%$ after 75 years [3].

Meningiomas are more commonly reported in females (sex ratio 2-4:1) [4] and the incidence increases with age. The only proved extrinsic risk factors are radiation therapy and hormone intake, both with a dose-response relationship. Meningiomas can arise in all irradiated patients, even after low dose ionizing radiation like for tinea capitis treatment, but the risk is higher in patients exposed to high-dose radiotherapy: in children exposed to cranial radiotherapy, the incidence of meningioma is $5.6 \%$ by age $40[5,6]$. Hormones such as cyproterone acetate, a synthetic steroidal antiandrogen, increase the risk of developing and growing meningiomas up to 11 times [7-9]. Other suspected risk factors, like cell phone exposure, are not confirmed in large cohorts [10].

\section{Classification and prognosis}

\subsection{Meningioma grading}

Meningiomas are classified according to the 2000/2007/2016 WHO classifications based on their histological appearance, divided into 15 subtypes, and on various aggressiveness criteria (mitoses, necrosis, cellular aspect), that are detailed in Table 1 [11]. Overall, more than $80 \%$ are grade I benign tumors [4], while atypical grade II include $4-15 \%$ of meningiomas and malignant grade III account for $1-3 \%$ of cases [12]. In the 2004-2010 US cohort of primary brain tumors, the proportion of each grade was $94.6,4.2$, and $1.2 \%$, respectively [13].

Grades II and III meningiomas are diagnosed in younger patients (median age 57 year old) and are more common in male $[14,15]$ and can develop de novo or arise from low-grade meningiomas transformation; those tumors seem to constitute two different molecular subtypes, de novo meningiomas conferring a better survival [16].

Although histological grading is the current gold standard in terms of diagnosing and treating meningiomas, its relevance is a subject of debate. Tumors are very heterogeneous within each grade, especially when grading relies on minor criteria, brain invasion or histologic aspect rather that mitotic count [17]. Moreover, some diagnostic criteria are vaguely defined and subject to a high interobserver bias [18-20]. In prospective clinical trials, when studying pathology concordance levels for meningioma classification and grading, there are respective concordance rates of $93.0,87.8$, and $93.6 \%$ for grades I-II-III, which is higher than those found in glioma pathology (maximum 74\%), but is still considered suboptimal; 
Table 1. Current histological criteria for grading meningiomas according to the 2016 WHO classification.

\begin{tabular}{ll}
\hline & Grade 1 - Benign \\
& Meningothelial \\
& Fibrous (fibroblastic) \\
& Transitional (mixed) \\
& Psammomatous \\
Angiomatous & Microcystic \\
& Secretory \\
& Lymphocyte rich \\
& Metaplastic \\
& Grade II - Atypical \\
\hline Clear cell & 4-19 mitotic figures/10 HPF \\
Or brain invasion \\
Or 3 minor criteria: \\
- Increased cellularity \\
- Small cell with high N/C ratio \\
& - Large and prominent nucleoli \\
& - Patternless or sheet-like growth \\
& - Foci of 'spontaneous' or geographic necrosis \\
\hline Rhabdoid & Grade III - Anaplastic \\
Papillary & $\geq 20$ mitotic figures/10 HPF \\
\hline
\end{tabular}

the authors suggest that education and clarification of subjective criteria, alongside biomarkers, will improve reproducibility [21]. Many discrepancies also arise from the evolution of histological classifications, that led to a dramatic increase of the proportion of grade II tumors, from $4.4 \%$ before 2000 up to $32.7 \%$ in 2006 [22,23]. In France, 13,038 meningiomas were histologically diagnosed between 2006 and 2010, among which $8.5 \%$ of atypical meningiomas but this number varies from 5 to $23 \%$ in other series and significantly increased after 2007 [2,24]. This has a considerable impact on therapeutic choices and clinical results and should be taken into account in retrospective series of patients, with particular attention paid to centralized histological review according to the 2016 WHO classification.

\subsection{Other classifications}

Although histological grading remains the gold standard in terms of diagnosis and treatment options, it is relevant to classify meningiomas according to other factors, such as the influence of hormone intake or the tumor location, as it may affect the treatments feasibility, and because the distribution of grading varies in these groups. Topographic description includes supra- or infra-tentorial or anterior, middle, and posterior fossa tumors, midline or lateral lesions, and invasion of other structures such as vessels or cranial nerves (cf. Figure 1). Meningiomas located in the anterior fossa, median middle fossa or anterior calvarium are more frequently grade I meningothelial or transitional meningiomas, whereas secretory

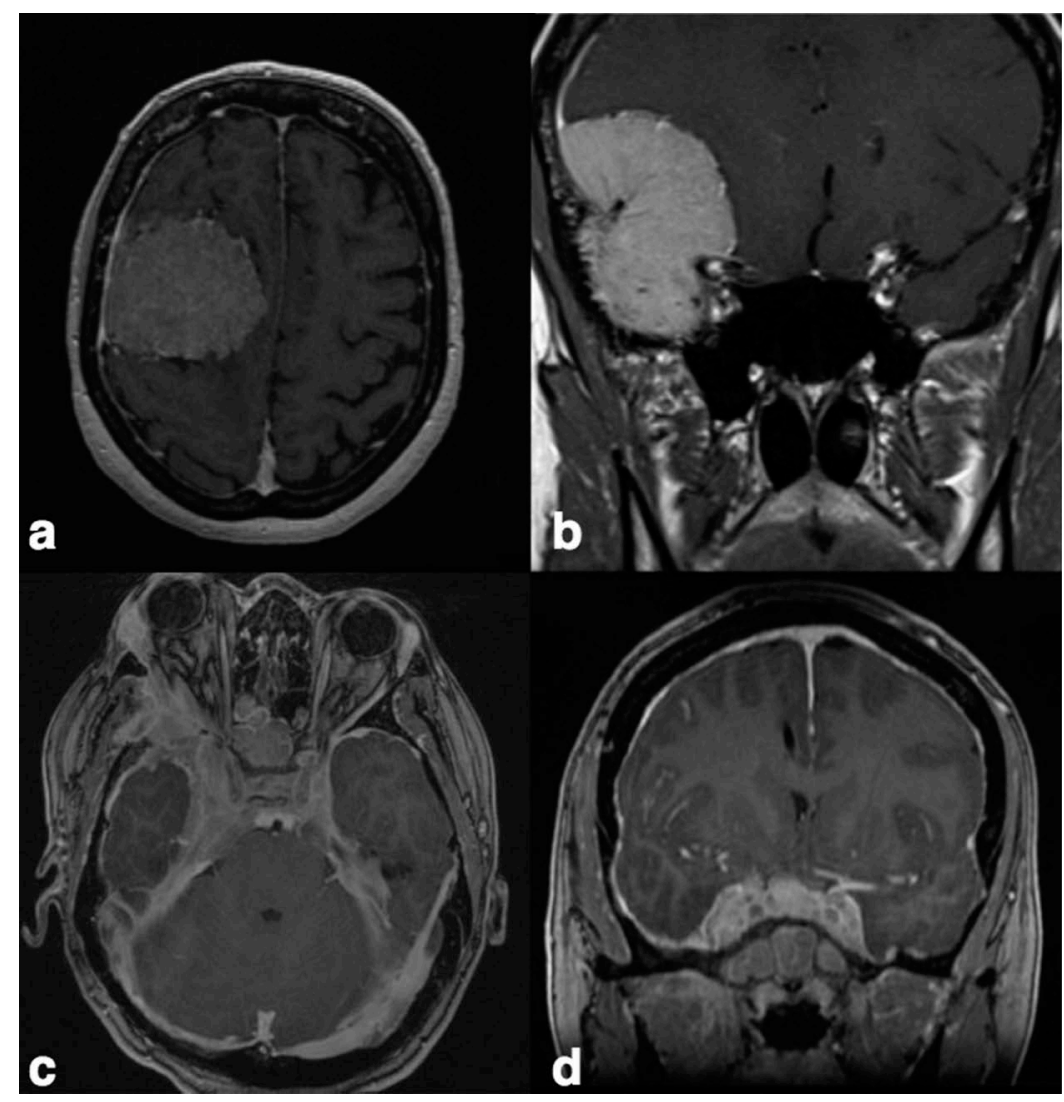

Figure 1. MRI of grade I meningiomas, T1-weighted gadolinium enhanced sequences. (a) This meningioma developing on the right frontal convexity can be treated surgically. (b) This right temporal base meningioma should be treated surgically because of its volume and accessibility. Total resection may not be possible because of the proximity of the middle cerebral artery. Radiotherapy or radiosurgery will be indicated in case of secondary regrowth. (c, d) This extensive skull base meningioma encloses both internal carotids, middle cerebral arteries, cranial nerves including optic nerves, and the sella turcica. Resection surgery is not possible and only a biopsy should be considered. In this particular case, the patient benefited from drilling of hyperostic bone to release the optic nerves, and her ovarian stimulation was stopped. She received proton beam radiotherapy. Systemic therapy would bring a significant clinical benefit by sparing the functional structures in the area. 
meningiomas more often develop in the lateral middle fossa, median posterior fossa as well as anterior fossa and median middle fossa $[25,26]$. Those subtypes are also associated with particular genetic profiles that are detailed below.

Recently, some authors have addressed the need for more reliable predictive markers by exploring the molecular profiles of meningiomas [27]. Recurrent mutations have been identified, but the main step toward a multilayered classification integrating clinical, histological, and biological data has been brought by an epigenetics study: Sahm et al. found that patients with meningioma could be divided into six DNA methylation groups, and that those groups were more accurately stratified for tumor behavior than the WHO classification [20].

\subsection{Clinical prognostic factors}

Even though meningiomas are benign tumors in most cases, patients have a shorter survival compared to a matched population. In 1998, the 5-year survival was $70 \%$ for the so-called benign meningiomas and 55\% for malignant ones [28], which is shorter than age-matched populations $[4,29]$. Nowadays, the 5- and 10-year survival rates are 65 and 50\% for grade II and III meningiomas [14,30]. Around $10 \%$ of grade I, $30-40 \%$ of grade II and $50-90 \%$ of grade III recur or progress after 10 years $[4,18]$. Prognostic factors include age, male gender, low Karnofsky performance status, high grade, high mitotic rate, subtotal surgical resection and involvement of the optic nerve
$[4,14,15,31,32]$. Somatic metastases are exceptional (0.1\%) [33] but isolated cases have been described for grade II and III recurrent meningiomas, in parasagittal locations that allow venous diffusion through the superior sagittal sinus [15,34]. Genetic and epigenetic subtypes seem to predict prognosis more accurately and are progressively becoming common practice for complex cases [20].

In brief, the most problematic meningiomas fall into two categories. First, those that develop on the skull base: although usually low grade, they enclose vasculo-nervous structures, posing a surgical challenge and causing functional and vital risks (cf. Figure 1). Second, grade II-III meningiomas, classically located at the skull convexity, that frequently recur, especially when venous invasion hinders complete surgical resection (cf. Figure 2).

\section{First-line treatments}

\subsection{Observation and symptomatic treatments}

The classical first-line treatment for all meningiomas is surgery. However, a wait-and-see strategy should always be considered when the clinical situation allows it, with regular clinical and MRI follow-up: predictive factors for observing rather than operating newly diagnosed meningiomas are a small diameter, below $2.5 \mathrm{~cm}$, the absence of neurological symptoms, calcifications on brain CT-scan and hyposignal intensity on

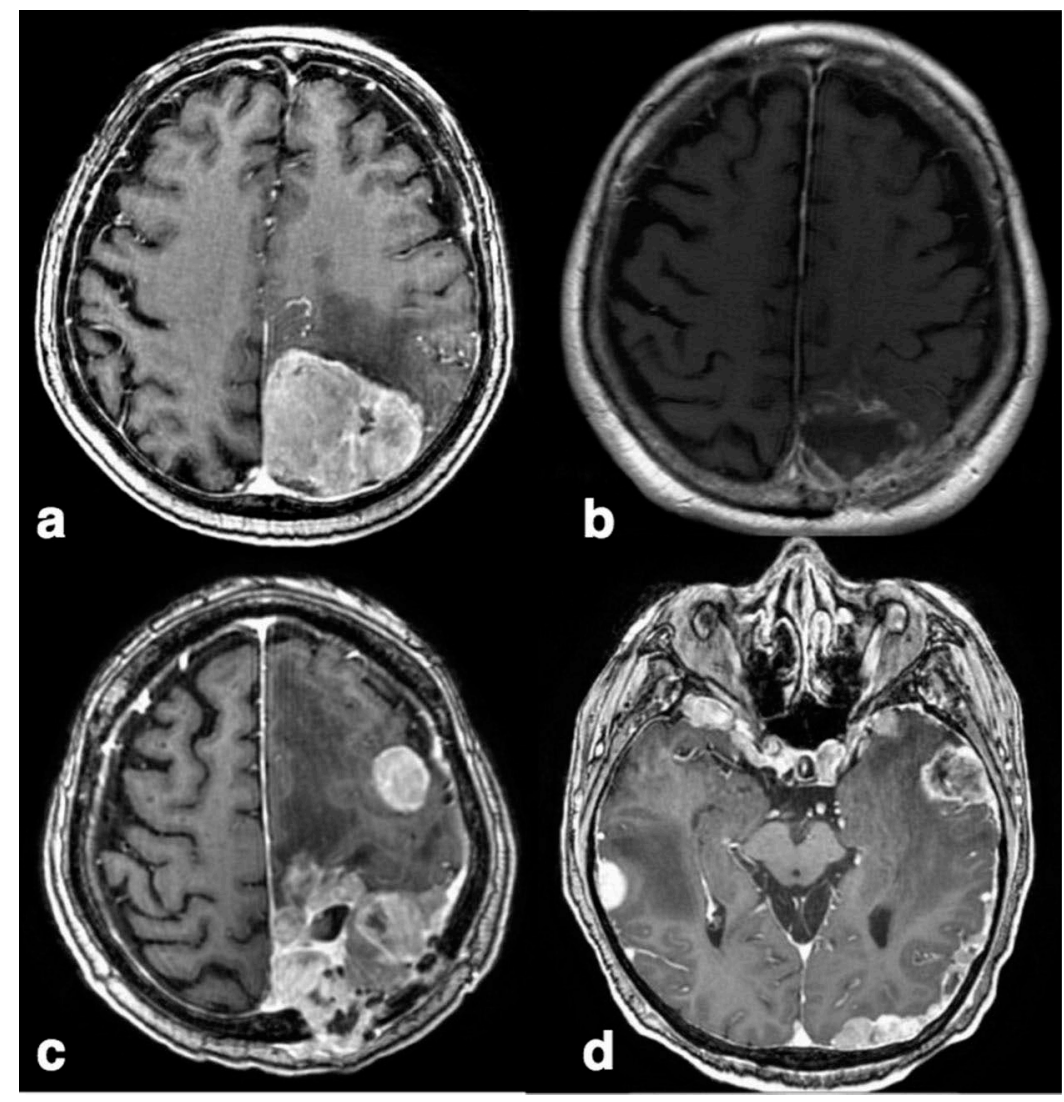

Figure 2. MRI of a patient with recurrent grade III meningioma, T1-weighted gadolinium enhanced sequences. (a) Initial presentation. The left occipital meningioma is amenable to surgical resection. (b) Postoperative MRI shows gross total resection of the tumor. (c) Despite repeated surgery and radiotherapy, the meningioma recurred locally recurrence with bone and skin invasion. (d) A few months later, MRI shows multinodular relapse with diffuse pachymeningeal thickening in both hemispheres. In those situations, global imaging follow-up is necessarily based on volumetric measures. This case underlines the need for systemic therapy in refractory patients. 
brain MRI [35]. Observation is classically not a long-term option for tumors $\geq 4 \mathrm{~cm}$, and for those with an initial growth rate of $\geq 20 \%$ /year for tumors $<2.5 \mathrm{~cm}$ and $\geq 1 \mathrm{~cm}^{3} /$ year for

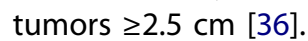

Careful questioning of the patients must look for exogenous hormone use (cyproterone acetate), sometimes prescribed for treating hirsutism, acne, seborrhea, alopecia or in transsexual patients. In those situations, withdrawing the hormone treatment is sometimes sufficient to stabilize and even decrease the tumor size [37-39]: some authors reported up to $80 \%$ volume reduction after one year of hormone disruption [40]. For the same reasons, meningiomas can also spontaneously decrease after pregnancy [41].

Symptomatic treatments include oral or intravenous steroids, that may help improve the mass effect by reducing peripheral edema to temporarily relieve the symptoms, for instance before surgery. Antiepileptic drugs are introduced only in epileptic patients, but preventive treatment is not recommended. Surgical removal helps achieving seizure freedom in $70 \%$ of preoperatively epileptic patients, but it can also induce epilepsy, in $12 \%$ of the preoperatively non-epileptic patients [42].

\subsection{Surgery}

During the last decades, the surgical techniques have evolved progressively with more recently the development of minimally invasive endoscopic transphenoidal approaches for skull base meningiomas [43]. However, after being very popular, it is already declining because serious local and neurological complications may arise, with cerebrospinal fluid leakage [44].

Depending on the location of the tumor and of the invasion of surrounding structures and brain parenchyma, total removal is not always possible. Post-operative Simpson grading based on the surgeon opinion, evaluates removal from grade 1 (complete) to 5 (simple biopsy) and allows, to predict symptomatic recurrence at 10 years, from 10 to $100 \%$ [45], regardless of the histological grade. Several authors have questioned this result since it was first published in 1957, especially in the absence of systematic postoperative MRI. It has been confirmed that, for grade II meningiomas for instance, Simpson 1 resection patients have a longer overall and progression free survival [15]. For grade III meningiomas selectively, the progression-free survival at 5 years is $28 \%$ after gross total resection alone, versus $0 \%$ after subtotal removal alone [46,47]. Although the results all tend to favor gross total resection, this goal should not affect the patients' immediate neurological status, and combined strategies could be used to maximize progression-free survival while reducing the neurological risks.

\subsection{Radiation therapy as a first-line treatment}

Although surgery remains central because it can relieve the tumor mass effect and establish histological diagnosis, radiation therapy has become a first-line option for some meningiomas, particularly skull base lesions encasing vasculo-nervous structures such as the optic nerve sheath or the cavernous sinus [48]. Radiation treatment alone may be offered if imaging is typical and surgery not feasible. Fractioned and hypofractioned stereotactic radiotherapy and radiosurgery, single or multiple doses, have all proved beneficial to patients with a high tumor control rate varying from 85 to $100 \%$ at 5 years $[49,50]$. Those results, alongside with radiation-induced toxicity, make these treatments particularly relevant for intractable tumors $<3 \mathrm{~cm}$. Side-effects of stereotactic radiotherapy for small tumors are mild [51,52], but cases of radionecrosis have been reported, and pituitary function must also be monitored after skull base irradiation [53].

\subsection{Adjuvant treatment - radiotherapy indications}

Grade I meningiomas are usually treated by surgery or radiosurgery alone, and adjuvant radiotherapy is only necessary for growing remnants [54]. On the contrary, grade II and III meningiomas are aggressive tumors with higher recurrence rates, up to, respectively, $30-40 \%$ and $50-80 \%$ at 5 years, versus $10 \%$ for grade I $[12,15,19,31,55]$; adjuvant radiation therapy of the tumor zone might be beneficial even after gross total resection $[15,19,56]$. Recurrences can be local or affect all the meninges [57]. Several types of radiation therapy have been proposed and must be chosen depending of the grade, size and location of the tumor. In case of a limited tumor, radiosurgery or stereotactic radiotherapy in single or multiple doses is appropriate (Figure 3). For recurrent, multiple or extensive lesions, external beam radiotherapy is the standard technique, with a dose of up to $70 \mathrm{~Gy}$ for grade II-III meningiomas, whether 3D conformal radiotherapy or intensity-modulated radiation therapy with or without tomotherapy [54]. Proton radiotherapy is also beneficial, and can be used in combination with photon radiotherapy [58].

For grade III tumors, it is established that adjuvant radiation improves long-term control and overall survival $[15,19,46]$, even after total gross removal: the progression-free survival at 5 years is improved from $28 \%$ for total removal alone, versus $57 \%$ for total removal with adjuvant radiotherapy [46]. In contrast, there is conflicting evidence for its role in grade II meningiomas. It has been shown that radiation therapy improves overall and progression-free survival when the tumor has been sub-totally removed [59], but not after total gross resection [60]. Moreover, retrospective studies did not always find a beneficial effect, and no prospective trial has been published yet $[15,30,51,61]$. Indeed, reported side effects of radiotherapy and radiosurgery are usually mild but there is also evidence that radiation increases the risk of malignant transformation [62].

Three comparable prospective trials started in the past years: The European Organization for Research and Treatment of Cancer (EORTC) led a randomized phase II study in 2008-2013, evaluating adjuvant postoperative radiation therapy versus observation in patients with newly diagnosed grade II or grade III meningiomas (NCT00626730, Switzerland). This trial was interrupted because of major protocol deviations and lack of inclusions [63]. The second one is the American RTOG 0539 (NCT00895622) trial concerning observation for lowrisk meningiomas and radiotherapy for intermediate and highrisk meningiomas, included 244 patients and is still ongoing. Initial outcomes after 3 years show that patients with completely resected grade II and recurrent grade I treated with postoperative radiotherapy experience a $96 \%$ rate of progression free survival [64]. Last, a phase II randomized controlled trial started in 2015 in the UK (ROAM/EORTC-1308) [65] comparing 


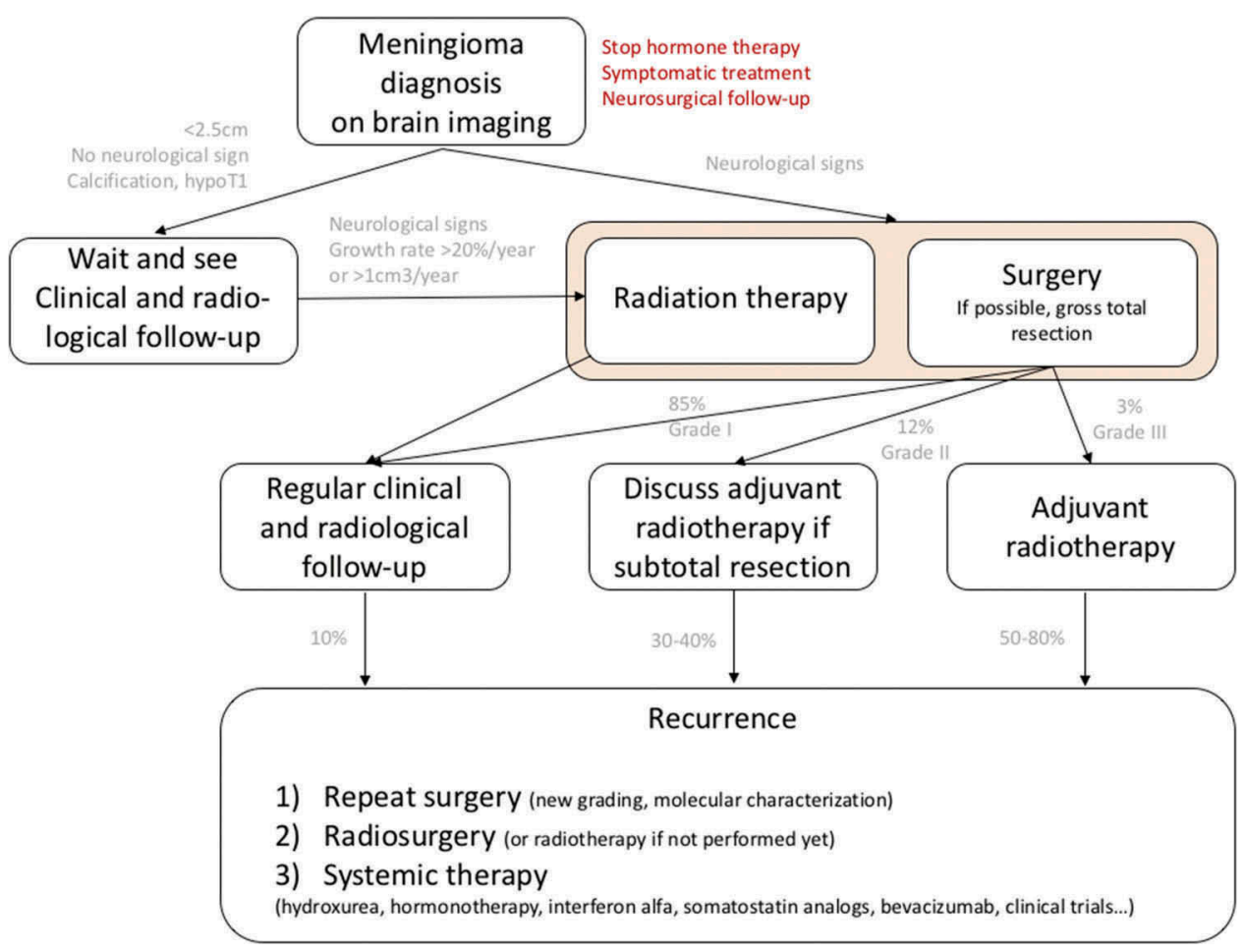

Figure 3. Care algorithm for patients with intracranial meningiomas.

radiation (60 Gy in 30 fractions) versus observation following surgical resection of atypical meningioma.

In clinical practice, therapeutic decision varies in different centers. In the UK, Germany, and France, 59, 74, and $80 \%$ of neurosurgeons, respectively, treat patients with radiotherapy after subtotal grade II removal, while $45-60 \%$ of neurosurgeons advise immediate adjuvant radiotherapy after grade II gross total resection $[15,66,67]$. These numbers have changed dramatically before and after 2000 , reflecting the changes in grade II meningiomas aggressiveness [23].

\subsection{Follow up}

There is no solid data about the best follow-up schedule. The European Association of Neuro-Oncology offers recommendations based on expert consensus [18]. For newly diagnosed asymptomatic meningiomas, a first MRI with injection may be considered after 6 months, then annually unless symptoms appear. After surgery, MRI should be performed ideally $<48 \mathrm{~h}$ to document the extent of resection. For completely resected grade I meningiomas, MRI controls can be performed annually for 5 years then every 2 years. After incomplete resection, a more vigilant follow up could include MRI at 6 and 12 months, then annually. For grade II and III tumors, it is appropriate to perform MRI every 6 months for 5 years, then annually, or even every 3 months in case of rapidly evolving lesions. In order to avoid underestimating slow tumor growth on MRl, some systematic rules should be followed: always compare the new MRI to a reference MRI obtained just after surgical resection, or when the meningioma was discovered; measure meningiomas with volumes rather than with planar dimensions [57].

\section{Medical treatments for recurrent meningiomas}

Long-term follow-up studies have shown that, even among the so-called completely resected tumors, up to $60 \%$ may recur after 15 years [19]. Usual treatments, as discussed nowadays by multidisciplinary teams, include repeating surgery and performing targeted radiosurgery in order to complete radiotherapy. Systemic therapies are chosen on an individual basis once surgical and radiation possibilities have been exhausted because of their limited efficacy. Classical chemotherapy schemes (temozolomide, irinotecan, doxorubicin, ifosfamide) have not proven efficient $[68,69]$. Hydroxyurea only has showed a stabilizing activity in some series [70-72], but this has not been consistently confirmed [73-75]. Some authors suggest that individual chemotherapy should be based on the expression of drug-resistant genes $[69,76]$. As a proof of concept, long-term efficacy of mitoxantrone and hydroxyurea has been reported in a specifically selected patient in whom mRNA analysis had predicted sensitivity to the chemotherapy [77]. The EORTC started a randomized trial in 2015 evaluating trabectedin, an alkylating agent classically used in soft-tissues sarcomas, in refractory recurrent grade II-grade III meningiomas (NCT02234050).

Hormonal treatments, either mifepristone or tamoxifen, have resulted in minor responses for some patients but without any impact on progression or survival [78]. Interferon-alpha helps stabilizing or diminishing recurrent meningiomas [79-82] and so do somatostatin analogs [68]. All these treatments have a limited and not highly repeatable effect. Combination of treatments may improve their efficacy, as tested in a currently recruiting prospective trial (NCT02333565, France) offering octreotide, a somatostatin analog, to enhance the inhibitory effect of everolimus, a mTOR inhibitor, in grade II-III and recurrent grade I meningiomas [83]. A phase II trial proposing a mTor 
inhibitor, vistusertib, in recurrent or progressive grade II-III meningiomas, started in 2017 (NCT03071874, USA).

Antiangiogenic treatments are increasingly used in clinical practice. Some molecules targeting vascular endothelial growth factor receptor, platelet-derived growth factor receptor, such as sunitinib and vatalanib, prolonged progressionfree intervals in patients with recurrent grade II and III meningiomas $[84,85]$. In particular, sunitinib was beneficial in a prospective phase II trial [86], and bevacizumab prolonged progression-free survival in retrospective studies [87,88], independently of molecular mutations, including NF2 [89]. An ongoing clinical trial (NCT01125046, USA) prospectively evaluates the efficacy of bevacizumab in recurrent or progressive meningiomas, and should be completed by 2018. In both cases, intracranial hemorrhage was the main adverse effect, in up to $20 \%$ of patients.

Immunotherapy may offer new therapeutic options. Highgrade meningiomas show PD-L1+ cells infiltration, which makes immune checkpoint inhibitors potentially beneficial treatments, like in mesotheliomas and pancreatic carcinomas [90]. Current clinical trials test anti-PD1 drugs pembrolizumab (NCT03279692) and nivolumab (NCT03173950) in recurrent or residual high-grade meningiomas.

\section{Molecular characterization and implications}

Molecular characterization of meningiomas has developed in the last decade and is already paving the way to new therapeutic approaches [91]. The most common genetic event is the inactivation of the NF2 tumor suppressor gene, found in 55\% of all meningiomas [25], and in $100 \%$ in patients with Neurofibromatosis type 2 who develop multiple meningiomas in addition to bilateral vestibular schwannoma [92]. Recently, mutations in genes such as TRAF7, AKT1, KLF4, SMO, PIK3R1, TERT, ARID1A, PIK3CA were identified, accounting altogether with NF2 for $80 \%$ of meningiomas [25,93-95]. Animal models developed in vivo in mice and in vitro lead to a better understanding of the molecular mechanisms that initiate tumorigenesis (in particular, NF2 mutation) and explain malignant transformation (added CDKN2A alteration for instance) [96-99].

Molecular characterization is being increasingly used for developing and directly testing therapeutic hypothesis, as show the inclusion criteria of some of the 23 ongoing clinical trials for patients with meningiomas indexed on clinicaltrials. org. Some molecular findings are particularly relevant either because they correlate with specific locations of meningiomas and may be targeted in case of surgically intractable tumors, or because specific treatments already exist. For instance, because of SMO role in embryogenesis, SMO mutations have been identified mainly in skull base meningiomas [26,93], and TRAF7 mutations in aggressive midline meningiomas [100]. Analyzing the molecular profile of these meningiomas, either after surgical sampling or maybe, one day, based on their MRI aspect, could allow safer individual targeted treatments, such as SMO inhibitor sonidegib.

An ongoing phase II clinical trial (NCT02523014, USA) [95] offers a new approach, where therapies are administered based on precise molecular identification of target mutations: patients with progressive meningiomas harboring NF2 mutations receive a FAK inhibitor (classically used in mesotheliomas [101]), whereas those harboring SMO mutations receive vismodegib, which has proved beneficial in patients with PTCH1-SMO mutated medulloblastomas [102]. This basket trial opens the doors to treatments based on molecular profiling, rather than histology.

\section{Expert commentary}

Meningioma is the most frequent primary tumor in the central nervous system, the most frequent pathology treated by neurosurgeons for decades. If surgical resection remains the firstline treatment in symptomatic patients, radiation therapy may be a useful alternative or adjuvant treatment, either for difficult skull base cases or aggressive variants.

So far, the recently modified histological WHO classification serves as a basis for therapeutic decision: while grade I tumors seldom recur and can be simply monitored after surgery, patients with high-grade meningiomas should benefit from postoperative radiation therapy. Grade II meningiomas constitute a highly heterogeneous group of tumors, precluding straightforward therapeutic guidelines. Those guidelines are highly debated because of the weak reliability of histological criteria and finding molecular predictive factors is at the heart of current research.

Recurrent meningioma is a complex condition in which surgical and radiosurgical options are to be considered first. Pharmacological alternatives are very diverse but none of them has proved systematically beneficial. Many clinical trials, sometimes including molecular characterization of the tumor, are ongoing and should be proposed to patients with intractable tumors whenever possible.

\section{Five-year view}

Molecular characterization of meningiomas has led to a new definition of meningiomas, based not on their histological aspect but on molecular changes affecting most of the time NF2, but also $A K T, S M O, T R A F 7 .$. Interestingly, these mutations seem to be associated with particular locations or aggressiveness subtypes, for instance SMO mutations are associated with olfactory groove recurrent meningiomas. Most of them can be targeted by molecules that have been developed in other clinical contexts, such as vismodegib for SMO-mutated lesions. In parallel, the epigenetic approach to meningiomas has recently led to a new classification based on methylation profiles that seems to predict very accurately tumor behavior. Although still beginning, those complementary perspectives pave the way to an integrated classification, associating clinical, histological, and molecular factors.

Based on those new subtypes, innovative therapeutic approaches are already being tested actively in animal models [99] and in several ongoing clinical trials [91]. Individual treatments rely on individual molecular profiles, each of them constituting a rare event. That is why recruiting patients for prospective trials is difficult and why all specialists confronted with highly complicated cases of meningiomas should try and offer their patients' molecular profiling, if possible as part of a clinical trial. That may allow the development of precise effective targeted therapy for patients currently in complete treatment failure. 


\section{Key issues}

- With 5 cases $/ 100,000$ individuals a year, meningiomas are the most frequent primary tumor in the central nervous system. Its incidence increases with age.

- The WHO 2016 histological classification, based on the mitoses rate combined with subjective histological criteria, defines 3 grades of meningiomas, $80 \%$ being Grade I and, $15 \%$ and $2 \%$ being Grade II and III respectively.

- A wait and see strategy can often be chosen first, based on clinical and radiological observation alongside cessation of hormonal therapy (cyproterone acetate).

- Grade I meningiomas are classically cured by complete surgical resection, whereas grade II and III tumors include a continuum of more aggressive tumors that often require adjuvant treatment, either radiotherapy or stereotactic radiosurgery. Systemic treatments are not currently a firstline option.

- Basing therapeutic decisions on purely histological criteria is problematic because of the vagueness of the classification that leads to heterogeneity of so-called high grades meningiomas and high inter-observer bias. Molecular classifications, particularly DNA methylation profiles, might stratify for tumor behavior more accurately and improve the basis for decision making of additional therapy after surgery.

- New approaches combine the use of classical chemotherapy molecules (hydroxyurea, tamoxifen) and targeted treatments (anti-angiogenic molecules such as sunitinib or bevacizumab, immunotherapy, or FAK-inhibitors) depending on the molecular profile of the tumor.

- Several prospective ongoing trials include patients with recurrent or progressive meningiomas for targeted therapies.

\section{References}

Papers of special note have been highlighted as of interest (•) to readers.

1. Riemenschneider MJ, Perry A, Reifenberger G. Histological classification and molecular genetics of meningiomas. Lancet Neurol. 2006;5:1045-1054.

2. Zouaoui S, Darlix A, Rigau V, et al. Descriptive epidemiology of 13,038 newly diagnosed and histologically confirmed meningiomas in France: 2006-2010. Neurochirurgie. 2015 Jun 11. [Epub ahead of print].

- The largest cohort of histologically proven meningiomas, describing epidemiology and prognosis in detail.

3. Vernooij MW, Ikram MA, Tanghe HL, et al. Incidental findings on brain MRI in the general population. N Engl J Med. 2007;357:18211828.

4. Saraf S, McCarthy BJ, Villano JL. Update on meningiomas. Oncologist. 2011;16:1604-1613.

5. Ron E, Modan B, Boice JDJ, et al. Tumors of the brain and nervous system after radiotherapy in childhood. $N$ Engl J Med. 1988;319:1033-1039.

6. Bowers DC, Moskowitz CS, Chou JF, et al. Morbidity and mortality associated with meningioma after cranial radiotherapy: a report from the childhood cancer survivor study. JCO. 2017;35:1570-1576.

7. Gazzeri R, Galarza M, Gazzeri G. Growth of a meningioma in a transsexual patient after estrogen-progestin therapy. N Engl J Med. 2007;357:2411-2412.

8. Claus EB, Black PM, Bondy ML, et al. Exogenous hormone use and meningioma risk: what do we tell our patients? Cancer. 2007;110:471-476.
9. Gil M, Oliva B, Timoner J, et al. Risk of meningioma among users of high doses of cyproterone acetate as compared with the general population: evidence from a population-based cohort study. $\mathrm{Br} \mathrm{J}$ Clin Pharmacol. 2011;72:965-968.

10. Carlberg M, Hardell L. Pooled analysis of Swedish case-control studies during 1997-2003 and 2007-2009 on meningioma risk associated with the use of mobile and cordless phones. Oncol Rep. 2015;33:3093-3098.

11. Louis DN, Perry A, Reifenberger G, et al. The 2016 World Health Organization classification of tumors of the central nervous system: a summary. Acta Neuropathol. 2016;131:803-820.

- Describes the 2016 WHO criteria that currently serve as a basis for prognosis and decision-making for treatment.

12. Perry A, Brat DJ. Practical surgical neuropathology: a diagnostic approach. Amsterdam, The Netherlands: Elsevier Health Sciences; 2010.

13. Kshettry VR, Ostrom QT, Kruchko C, et al. Descriptive epidemiology of World Health Organization grades II and III intracranial meningiomas in the United States. Neuro Oncol. 2015;17:1166-1173.

14. Pasquier D, Bijmolt S, Veninga T, et al. Atypical and malignant meningioma: outcome and prognostic factors in 119 irradiated patients. A multicenter, retrospective study of the rare cancer network. Int J Radiat Oncol Biol Phys. 2008;71:1388-1393.

15. Durand A, Labrousse F, Jouvet A, et al. WHO grade II and III meningiomas: a study of prognostic factors. J Neurooncol. 2009;95:367-375.

16. Moliterno J, Cope WP, Vartanian ED, et al. Survival in patients treated for anaplastic meningioma. J Neurosurg. 2015;123:23-30.

17. Vaubel RA, Chen SG, Raleigh DR, et al. Meningiomas with rhabdoid features lacking other histologic features of malignancy: a study of 44 cases and review of the literature. J Neuropathol Exp Neurol. 2016;75:44-52.

18. Goldbrunner R, Minniti G, Preusser M, et al. EANO guidelines for the diagnosis and treatment of meningiomas. Lancet Oncol. 2016;17: e383-e391.

19. Rogers L, Barani I, Chamberlain M, et al. Meningiomas: knowledge base, treatment outcomes, and uncertainties. A RANO review. J Neurosurg. 2015;122:4-23.

- The current European guidelines about meningioma treatments.

20. Sahm F, Schrimpf D, Stichel D, et al. DNA methylation-based classification and grading system for meningioma: a multicentre, retrospective analysis. Lancet Oncol. 2017;18:682-694.

- The recent global article outlining the contribution of DNA methylation to predict meningioma prognosis. The authors find that their methylation groups stratify tumors better than current histological classification.

21. Rogers CL, Perry A, Pugh S, et al. Pathology concordance levels for meningioma classification and grading in NRG oncology RTOG trial 0539. Neuro Oncol. 2016;18:565.

22. Combs SE, Schulz-Ertner D, Debus J, et al. Improved correlation of the neuropathologic classification according to adapted world health organization classification and outcome after radiotherapy in patients with atypical and anaplastic meningiomas. Int J Radiat Oncol Biol Phys. 2011;81:1415-1421.

23. Pearson BE, Markert JM, Fisher WS, et al. Hitting a moving target: evolution of a treatment paradigm for atypical meningiomas amid changing diagnostic criteria. Neurosurg Focus. 2008;24:E3.

24. Hwang KL, Hwang WL, Bussière MR, et al. The role of radiotherapy in the management of high-grade meningiomas. Chin Clin Oncol [Internet]. 2017;6. [cited 2017 Oct 2]. Available from: http://cco. amegroups.com/article/view/15550

25. Yuzawa S, Nishihara H, Tanaka S. Genetic landscape of meningioma. Brain Tumor Pathol. 2016;33:237-247.

26. Boetto J, Bielle F, Sanson M, et al. SMO mutation status defines a distinct and frequent molecular subgroup in olfactory groove meningiomas. Neuro Oncol. 2017;19:345-351.

27. Galani V, Lampri E, Varouktsi A, et al. Genetic and epigenetic alterations in meningiomas. Clin Neurol Neurosurg. 2017;158:119-125. 
28. McCarthy BJ, Davis FG, Freels S, et al. Factors associated with survival in patients with meningioma. J Neurosurg. 1998;88:831-839.

29. Sankila R, Kallio M, Jääskeläinen J, et al. Long-term survival of 1986 patients with intracranial meningioma diagnosed from 1953 to 1984 in Finland. Comparison of the observed and expected survival rates in a population-based series. Cancer. 1992;70:1568-1576.

30. Kaur G, Sayegh ET, Larson A, et al. Adjuvant radiotherapy for atypical and malignant meningiomas: a systematic review. Neuro Oncol. 2014;16:628-636.

31. Kim D, Niemierko A, Hwang WL, et al. Histopathological prognostic factors of recurrence following definitive therapy for atypical and malignant meningiomas. J Neurosurg. 2017 Jun 16:1-10. [Epub ahead of print].

32. Stafford SL, Perry A, Suman VJ, et al. Primarily resected meningiomas: outcome and prognostic factors in 581 Mayo Clinic patients, 1978 through 1988. Mayo Clin Proc. 1998;73:936-942.

33. Kessler RA, Garzon-Muvdi T, Yang W, et al. Metastatic atypical and anaplastic meningioma: a case series and review of the literature. World Neurosurg. 2017;101:47-56.

34. Paix A, Waissi W, Antoni $D$, et al. Visceral and bone metastases of a WHO grade 2 meningioma: a case report and review of the literature. Cancer Radiother. 2017;21:55-59.

35. Lee EJ, Park JH, Park ES, et al. "Wait-and-see" strategies for newly diagnosed intracranial meningiomas based on the risk of future observation failure. World Neurosurg. 2017;107:604-611.

36. Lee EJ, Kim JH, Park ES, et al. A novel weighted scoring system for estimating the risk of rapid growth in untreated intracranial meningiomas. J Neurosurg. 2017;127:971-980.

37. Cebula $H$, Pham TQ, Boyer $P$, et al. Regression of meningiomas after discontinuation of cyproterone acetate in a transsexual patient. Acta Neurochir (Wien). 2010;152:1955-1956.

38. Botella C, Coll G, Lemaire J-J, et al. [Intra cranial meningiomas and long term use of cyproterone acetate with a conventional dose in women. A report of two cases of tumor decrease after treatment withdrawal]. Neurochirurgie. 2015;61:339-342.

39. Bernat AL, Oyama K, Hamdi S, et al. Growth stabilization and regression of meningiomas after discontinuation of cyproterone acetate: a case series of 12 patients. Acta Neurochir (Wien). 2015;157:1741-1746.

40. Kalamarides M, Peyre M. Dramatic shrinkage with reduced vascularization of large meningiomas after cessation of progestin treatment. World Neurosurg. 2017;101:814.e7-814.e10.

41. Kerschbaumer J, Freyschlag CF, Stockhammer G, et al. Hormonedependent shrinkage of a sphenoid wing meningioma after pregnancy: case report. J Neurosurg. 2016;124:137-140.

42. Englot DJ, Magill ST, Han SJ, et al. Seizures in supratentorial meningioma: a systematic review and meta-analysis. J Neurosurg. 2016;124:1552.

43. Zada G, Başkaya MK, Shah MV. Introduction: surgical management of skull base meningiomas. Neurosurg Focus. 2017;43:Intro.

44. Muskens IS, Briceno V, Ouwehand TL, et al. The endoscopic endonasal approach is not superior to the microscopic transcranial approach for anterior skull base meningiomas-a meta-analysis. Acta Neurochir (Wien). 2018;160(1):59-75.

45. Simpson $D$. The recurrence of intracranial meningiomas after surgical treatment. J Neurol Neurosurg Psychiatr. 1957;20:22-39.

46. Dziuk TW, Woo S, Butler EB, et al. Malignant meningioma: an indication for initial aggressive surgery and adjuvant radiotherapy. J Neurooncol. 1998;37:177-188.

47. Sughrue ME, Kane AJ, Shangari G, et al. The relevance of Simpson grade I and II resection in modern neurosurgical treatment of World Health Organization grade I meningiomas. J Neurosurg. 2010;113:1029-1035.

48. Prabhu VC, Melian E, Germanwala AV, et al. Cranial base meningiomas. World Neurosurg [Internet]. 2017. cited 2017 Oct 16. Available from http://www.sciencedirect.com/science/article/pii/S1878875017317254

49. Starke RM, Przybylowski CJ, Sugoto M, et al. Gamma Knife radiosurgery of large skull base meningiomas. J Neurosurg. 2015;122:363-372.

50. Patibandla MR, Lee -C-C, Sheehan J. Stereotactic radiosurgery of central skull base meningioma's - volumetric evaluation and longterm outcomes. World Neurosurg. 2017;108:176-184.
51. Cho M, Joo J-D, Kim IA, et al. The role of adjuvant treatment in patients with high-grade meningioma. J Korean Neurosurg Soc. 2017;60:527-533.

52. Valery CA, Faillot M, Lamproglou I, et al. Grade II meningiomas and Gamma Knife radiosurgery: analysis of success and failure to improve treatment paradigm. J Neurosurg. 2016;125:89-96.

53. Minniti G, Clarke E, Cavallo L, et al. Fractionated stereotactic conformal radiotherapy for large benign skull base meningiomas. Radiat Oncol. 2011;6:36.

54. Noël G, Renard A, Valéry C, et al. [Role of radiotherapy in the treatment of cerebral meningiomas]. Cancer Radiother. 2001;5:217-236.

55. Palma L, Celli P, Franco C, et al. Long-term prognosis for atypical and malignant meningiomas: a study of 71 surgical cases. J Neurosurg. 1997;86:793-800.

56. Walcott BP, Nahed BV, Brastianos PK, et al. Radiation treatment for WHO grade II and III meningiomas. Front Oncol [Internet]. 2013;3. cited 2017 Oct 2. Available from: https://www.ncbi.nlm.nih.gov/ pmc/articles/PMC3759290/

57. Peyre $M$, Zanello $M$, Mokhtari $K$, et al. Patterns of relapse and growth kinetics of surgery- and radiation-refractory meningiomas. J Neurooncol. 2015;123:151-160.

- Shows different patterns of evolution of atypical and anaplastic meningiomas, and gives a global indication of what could be expected in rare, aggressive cases.

58. Boskos C, Feuvret L, Noel G, et al. Combined proton and photon conformal radiotherapy for intracranial atypical and malignant meningioma. Int J Radiat Oncol Biol Phys. 2009;75:399-406.

59. Park $\mathrm{HJ}$, Kang $\mathrm{H}-\mathrm{C}$, Kim IH, et al. The role of adjuvant radiotherapy in atypical meningioma. J Neurooncol. 2013;115:241-247.

60. Graffeo CS, Leeper HE, Perry A, et al. Revisiting adjuvant radiotherapy after gross total resection of World Health Organization grade II meningioma. World Neurosurg. 2017;103:655-663.

61. Stessin AM, Schwartz A, Judanin G, et al. Does adjuvant externalbeam radiotherapy improve outcomes for nonbenign meningiomas? A Surveillance, Epidemiology, and End Results (SEER)-based analysis. J Neurosurg. 2012;117:669-675.

62. Yamanaka R, Hayano A, Kanayama T. Radiation-induced meningiomas: an exhaustive review of the literature. World Neurosurg. 2017;97:635.e8-644.e8.

63. Coskun M, Straube W, Hurkmans CW, et al. Quality assurance of radiotherapy in the ongoing EORTC 22042-26042 trial for atypical and malignant meningioma: results from the dummy runs and prospective individual case reviews. Radiat Oncol. 2013;8:23.

64. ASTRO 2015: patients with intermediate-risk meningiomas who receive postoperative radiotherapy have a $96 \% 3$-year progression-free survival - the ASCO post [Internet]. [cited 2017 Oct 12]. Available from: http://www.ascopost.com/News/33973

65. Jenkinson MD, Javadpour M, Haylock BJ, et al. The ROAM/EORTC1308 trial: radiation versus observation following surgical resection of atypical meningioma: study protocol for a randomised controlled trial. Trials. 2015;16:519.

66. Marcus HJ, Price SJ, Wilby M, et al. Radiotherapy as an adjuvant in the management of intracranial meningiomas: are we practising evidence-based medicine? Br J Neurosurg. 2008;22:520-528.

67. Simon M, Boström J, Koch $P$, et al. Interinstitutional variance of postoperative radiotherapy and follow up for meningiomas in Germany: impact of changes of the WHO classification. J Neurol Neurosurg Psychiatr. 2006;77:767-773.

68. Chamberlain MC, Barnholtz-Sloan JS. Medical treatment of recurrent meningiomas. Expert Rev Neurother. 2011;11:1425-1432.

69. Sioka C, Kyritsis AP. Chemotherapy, hormonal therapy, and immunotherapy for recurrent meningiomas. J Neurooncol. 2009;92:1-6.

70. Newton HB. Hydroxyurea chemotherapy in the treatment of meningiomas. Neurosurg Focus. 2007;23:E11.

71. Fuentes S, Chinot O, Dufour H, et al. [Hydroxyurea treatment for unresectable meningioma]. Neurochirurgie. 2004;50:461467.

72. Hahn BM, Schrell UMH, Sauer R, et al. Prolonged oral hydroxyurea and concurrent $3 \mathrm{~d}$-conformal radiation in patients with progressive or 
recurrent meningioma: results of a pilot study. J Neurooncol. 2005;74:157-165.

73. Mason WP, Gentili F, Macdonald DR, et al. Stabilization of disease progression by hydroxyurea in patients with recurrent or unresectable meningioma. J Neurosurg. 2002;97:341-346.

74. Loven D, Hardoff R, Sever ZB, et al. Non-resectable slow-growing meningiomas treated by hydroxyurea. J Neurooncol. 2004;67:221-226.

75. Weston GJ, Martin AJ, Mufti GJ, et al. Hydroxyurea treatment of meningiomas: a pilot study. Skull Base. 2006;16:157-160.

76. Andersson U, Malmer B, Bergenheim AT, et al. Heterogeneity in the expression of markers for drug resistance in brain tumors. Clin Neuropathol. 2004;23:21-27.

77. Kunishio K, Kobayashi K, Kagawa M, et al. [A case of malignant meningioma treated by individual adjuvant chemotherapy based on the mRNA expression of drug-resistance gene]. Gan To Kagaku Ryoho. 2007;34:265-268.

78. Ji Y, Rankin C, Grunberg S, et al. Double-blind phase III randomized trial of the antiprogestin agent mifepristone in the treatment of unresectable meningioma: SWOG S9005. J Clin Oncol. 2015;33:4093-4098.

79. Wöber-Bingöl $C$, Wöber $C$, Marosi $C$, et al. Interferon-alfa-2b for meningioma. Lancet. 1995;345:331.

80. Kaba SE, DeMonte F, Bruner JM, et al. The treatment of recurrent unresectable and malignant meningiomas with interferon alpha-2B. Neurosurgery. 1997;40:271-275.

81. Chamberlain MC, Glantz MJ. Interferon-alpha for recurrent World Health Organization grade 1 intracranial meningiomas. Cancer. 2008;113:2146-2151.

82. Chamberlain MC. IFN- $\alpha$ for recurrent surgery- and radiation-refractory high-grade meningioma: a retrospective case series. CNS Oncol. 2013;2:227-235.

83. Graillon T, Defilles C, Mohamed A, et al. Combined treatment by octreotide and everolimus: octreotide enhances inhibitory effect of everolimus in aggressive meningiomas. J Neurooncol. 2015;124:33-43.

84. Raizer JJ, Grimm SA, Rademaker A, et al. A phase II trial of PTK787/ZK 222584 in recurrent or progressive radiation and surgery refractory meningiomas. J Neurooncol. 2014;117:93-101.

85. Vranic A, Peyre M, Kalamarides $M$. New insights into meningioma: from genetics to trials. Curr Opin Oncol. 2012;24:660-665.

86. Kaley TJ, Wen $P$, Schiff D, et al. Phase II trial of sunitinib for recurrent and progressive atypical and anaplastic meningioma. Neuro Oncol. 2015;17:116-121.

87. Lou E, Sumrall AL, Turner S, et al. Bevacizumab therapy for adults with recurrent/progressive meningioma: a retrospective series. J Neurooncol. 2012;109:63-70.
88. Nayak L, Iwamoto FM, Rudnick JD, et al. Atypical and anaplastic meningiomas treated with bevacizumab. J Neurooncol. 2012;109:187-193.

89. Nunes FP, Merker VL, Jennings $D$, et al. Bevacizumab treatment for meningiomas in NF2: a retrospective analysis of 15 patients. PLoS One. 2013;8:e59941.

90. Han SJ, Reis G, Kohanbash G, et al. Expression and prognostic impact of immune modulatory molecule PD-L1 in meningioma. J Neurooncol. 2016;130:543-552.

91. Peyre M, Kalamarides M. Molecular genetics of meningiomas: building the roadmap towards personalized therapy. Neurochirurgie. 2014 Sep 19. [Epub ahead of print].

92. Goutagny S, Kalamarides M. Meningiomas and neurofibromatosis. J Neurooncol. 2010;99:341-347.

93. Clark VE, Erson-Omay EZ, Serin A, et al. Genomic analysis of nonNF2 meningiomas reveals mutations in TRAF7, KLF4, AKT1, and SMO. Science. 2013;339:1077-1080.

- This article describes the bases of meningioma genetics.

94. Abedalthagafi M, Bi WL, Aizer AA, et al. Oncogenic PI3K mutations are as common as AKT1 and SMO mutations in meningioma. Neuro Oncol. 2016;18:649-655.

95. Brastianos PK, Horowitz PM, Santagata S, et al. Genomic sequencing of meningiomas identifies oncogenic SMO and AKT1 mutations. Nat Genet. 2013;45:285.

96. Kalamarides $M$, Niwa-Kawakita $M$, Leblois $H$, et al. Nf2 gene inactivation in arachnoidal cells is rate-limiting for meningioma development in the mouse. Genes Dev. 2002;16:1060-1065.

97. Kalamarides M, Stemmer-Rachamimov AO, Takahashi M, et al. Natural history of meningioma development in mice reveals: a synergy of Nf2 and p16(Ink4a) mutations. Brain Pathol. 2008; 18:62-70

98. Peyre M, Salaud C, Clermont-Taranchon E, et al. PDGF activation in PGDS-positive arachnoid cells induces meningioma formation in mice promoting tumor progression in combination with Nf2 and Cdkn2ab loss. Oncotarget. 2015;6:32713-32722.

99. Kalamarides $M$, Peyre $M$, Giovannini M. Meningioma mouse models. J Neurooncol. 2010;99:325-331.

100. Peyre M, Feuvret $L$, Sanson $M$, et al. Diffuse midline skull base meningiomas: identification of a rare and aggressive subgroup of meningiomas. J Neurooncol. 2017;133:633-639.

101. Sulzmaier FJ, Jean C, Schlaepfer DD. FAK in cancer: mechanistic findings and clinical applications. Nat Rev Cancer. 2014;14:598-610.

102. Samkari A, White J, Packer R. SHH inhibitors for the treatment of medulloblastoma. Expert Rev Neurother. 2015;15:763-770. 